Proceeding of $3^{\text {rd }}$ International Conference on Engineering Mathematics and Physics Eph-1 iro

\author{
Military Technical College \\ Kobry Elkobbah, \\ Cairo, Egypt \\ May 16-18,2006
}

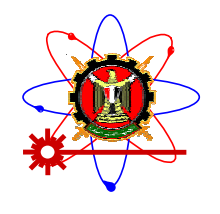

$3^{\text {nd }}$ International Conference on Engineering Mathematics and Physics

(ICMEP-3)

\title{
EXTINCTION-BASED VARIABLE OPTICAL ATTENUATOR IN InGaAsP/InP
}

\author{
ABDALLA S. ${ }^{*}$, NG S. ${ }^{* *}$, MOKHTAR M.M. ${ }^{*}$ and HASAN M.F. ${ }^{*}$
}

\begin{abstract}
An extinction-based compact InGaAsP/InP waveguide variable optical attenuator (VOA) with electrically modulated bend loss is demonstrated. Injection of carriers is utilized to modify the effective index of the waveguide bent section. The $p$-i-n semiconductor waveguide layer structure is discussed. Signal propagation at multiple bias conditions is simulated and the attenuator performance is calculated and compared to that of a straight waveguide attenuator. The device exhibits attenuation better than $15 \mathrm{~dB}$ for both TE and TM polarizations. 3-dB attenuation is achieved at an applied current of $\sim 12 \mathrm{~mA}$ which is less than half the current required by a comparable straight waveguide attenuator.
\end{abstract}

\section{KEY WORDS}

Bend loss attenuation, InGaAsP/InP, carrier injection, Variable optical attenuator.

\footnotetext{
*Egyptian Armed Forces.

** Department of Electronics, Carleton University, Canada.
} 
Proceeding of $3^{\text {rd }}$ International Conference on Engineering Mathematics and Physics Eph-1 ir

\section{INTRODUCTION}

Uses of optical attenuators in optical networks is widespread, such as reducing power output from an optoelectronic source, inline with amplifiers or add/drop multiplexers for channel equalization and at the detector as termination points [1]. According to requirements of present and future optical networks the desirable features of an optical attenuator are fast response, low power consumption, good reliability and easy integration with other optical components on the same chip. Waveguide attenuators can be implemented using either extinction of optical signal or optical path switching, such as an asymmetric Y-branch switch [2]. Extinctionbased attenuators are generally straight or bent waveguides, and the optical signal is attenuated through absorption [3] or radiation loss due to reduced confinement [4]. Attenuators based on extinction are generally more compact than those based on switching and can make use of existing connection waveguides in a planar lightwave circuit (PLC). Straight or bent waveguide attenuators have been proposed and successfully commercialized in polymer materials $[5,6]$ with actuation by the thermooptic effect. However, polymers are sensitive to heat, optical power, and are often birefringent. They exhibit very slow speed on the order of milliseconds [7] as their measured response times for thermo-optic actuation are long. Fabrication on III-V semiconductor allows waveguide attenuators to be integrated into PLCs and even incorporated into existing connection waveguides [8]. Potential actuation mechanisms in semiconductors include carrier injection, electroabsorption [3], and semiconductor optical amplifier (SOA) based modulation [9]. A preference for polarization and wavelength independence make electro-optic effect and SOA devices unattractive. Carrier injection is much faster than the thermo-optic effect and also wavelength and polarization insensitive. A straight waveguide modulator has been implemented in silicon using carrier injection to suppress the refractive index and modulate by "waveguide-vanishing" [4]. In polymers, several bent waveguide thermo-optic designs have been proposed that enhance modulation by adding bend loss $[7,10]$. We propose that the introduction of a simple bend in a waveguide carrier injection modulator implemented in semiconductor will similarly enhance attenuation and reduce actuation power.

\section{DEVICE DESCRIPTION}

The proposed bend loss attenuator consists of a straight waveguide section at the input followed by an S-bend leading to a straight output section. The S-bend is made from the intersection of arcs of two identical circular waveguides with radii $32 \mathrm{~mm}$ and width $5 \mu \mathrm{m}$ with a total length of $2 \mathrm{~mm}$. An electrode is placed over an $800 \mu \mathrm{m}$ part of the S-bend section as shown in Fig. 1. Note that the electrodes are only in electrical contact with the device where they overlap the waveguide ridges in the Sbend.

The layer structure is an InP/InGaAsP/InP p-i-n waveguide heterostructure grown on an $\mathrm{n}^{+}$-type InP substrate. Layers doping and dimensions are optimized by iterative calculations to assure single mode operation of the waveguide forming the optical attenuator. The layer structure consists of a $700 \mathrm{~nm}$ n-type InP buffer (Si-doped at $\left.5 \mathrm{e} 18 \mathrm{~cm}^{-3}\right), 300 \mathrm{~nm}$ undoped $\mathrm{InP}, 600 \mathrm{~nm} \operatorname{InGaAsP}$ waveguide core (Q=1.4 $\left.\mu \mathrm{m}\right), 300$ $\mathrm{nm}$ undoped InP, $200 \mathrm{~nm}$ p-type InP (Be-doped at $5 \mathrm{e} 17 \mathrm{~cm}^{-3}$ ), $10 \mathrm{~nm}$ InGaAsP etch- 
Proceeding of $3^{\text {rd }}$ International Conference on Engineering Mathematics and Physics Eph-1 irv

stop layer $(\mathrm{Q}=1.4 \mu \mathrm{m}), 400 \mathrm{~nm}$ p-type $\operatorname{lnP}\left(\right.$ Be-doped at $3 \mathrm{e} 17 \mathrm{~cm}^{-3}$ ) and a $200 \mathrm{~nm} \mathrm{p}$ type InGaAs cap (Be-doped at $1 \mathrm{e} 19 \mathrm{~cm}^{-3}$ ). The ridge waveguides were defined by a $5 \mu \mathrm{m}$ wide ridge with a $600 \mathrm{~nm}$ etch-depth as shown in Fig. 2.

\section{Theory of operation}

Vertical confinement of the light in the core layer is provided by the refractive index contrast between the high-index core and the lower-index cladding layers. Lateral confinement is provided by the effective index contrast between the ridge waveguide and its adjacent waveguide regions. Under forward bias, carriers are injected into the core causing a refractive index change resulting from the combination of plasma effect, bandfilling effect and bandgap shrinkage effect, the non-linear relation between the injected carrier's concentration and the refractive index change implies proper choice of operating conditions to achieve the required index change [11]. Beam propagation method (BPM) is used to simulate wave propagation in the optical attenuator. With no bias applied to the electrodes, the input optical power is guided to the output port with low propagation loss due to the large radius of curvature of the Sbend as shown in Fig. 3a. Forward biasing the attenuator with proper operating conditions causes a negative refractive index change of the core layer [12] which in turn reduces the effective index of the biased waveguide section. Optical confinement of the waveguide mode is therefore reduced allowing control of the optical attenuation in the waveguide bend. Scattered optical power through the forward biased device is shown in Fig. 3b. When the induced index change $\Delta n$ is equal to the difference in effective index between the ridge waveguide and the adjacent slab waveguide region, the optical field will no longer see a waveguide and attenuation is at its maximum.

\section{SIMULATION RESULTS}

Using the effective index method the bend loss attenuator performance is simulated with two dimensional BPM calculations. The effective index beneath the electrode is varied in steps of $\Delta n=-.001$. Fig. 4. shows the calculated optical power at the output port versus effective index change of the attenuator with an electrode length of 800 $\mu \mathrm{m}$ in comparison with a straight waveguide attenuator of the same overall length and same electrode length. A maximum value of $\Delta n$ was chosen to be -0.007 which is equal to the difference in effective index between the ridge waveguide and the adjacent slab waveguide region. At that value of $\Delta n$ there is no lateral optical confinement and light is scattered along the core layer of the chip. The presence of the bend in the device increases the attenuation by up to $5 \mathrm{~dB}$ compared to the straight waveguide attenuator for the same induced effective index change.

\section{EXPERIMENTAL RESULTS}

Metal-oxide chemical vapor deposition technique is used to grow the wafers. Chemically-Assisted-Ion-Beam-Etching (CAIBE) defined the ridge-waveguide on the 
p-layers and a final selective wet-etch completed the ridge by stopping at the thin InGaAsP etch stop layer. Electrodes are deposited using a metal stack of $500 \AA \mathrm{Ti}$ $1600 \AA \mathrm{Pt} / 5000 \AA \mathrm{Au}$. The back of the substrate is coated with $250 \AA \mathrm{Ni} / 550 \AA \mathrm{Ge}$ /2000A Au. Two sets of devices are prepared, the first set consists of bend waveguide attenuators and the other set consists of straight waveguide attenuators. Optical characterization of the VOA comprised coupling light from a broadband erbium doped fiber amplifier source $(\lambda=1530-1560 \mathrm{~nm})$ into the devices and collecting the output with a tapered fiber. The current is ramped from zero to $250 \mathrm{~mA}$ over several hundred nanoseconds, and the transmitted optical power is acquired using a fast photodetector and an oscilloscope. Experimental measurements of the attenuation versus applied current for bent and straight waveguide devices are shown in Fig. 5.

As with the BPM simulations, the presence of the bend in the device increased the attenuation by about $5 \mathrm{~dB}$ for the same applied current. An effective index modulation of -0.007 in the InGaAsP core corresponds to an applied current of about $0.055 \mathrm{~A}$ [12], so figures 4 and 5 can be directly compared. Both theoretical and experimental results show increased attenuation for the bent attenuator design. At high currents, the maximum measured attenuation is limited to $15 \mathrm{~dB}$, as seen in the inset of Fig. 5 . This saturation effect is due to light scattered in the biased section coupling back into the output waveguide port. By increasing the offset between the input and output waveguide ports this effect may be reduced, thus enhancing attenuation. An attenuator with a bend would require about half the current density for attenuation to $3 \mathrm{~dB}$ than a straight waveguide. To check polarization dependence both TE and TM modes are used in measurements for both straight and bent waveguides attenuators. It is clear that bent attenuators don't suffer from polarization dependence which is the main drawback of straight attenuators.

\section{SUMMARY}

We have designed, fabricated and measured a bend loss attenuator actuated by carrier injection with significant improvements in performance over a straight waveguide attenuator. Attenuation of $3 \mathrm{~dB}$ is achieved by an actuation current of $\sim 12$ $\mathrm{mA}$, which is less than half the current required by a comparable straight waveguide attenuator. The measured polarization dependence of the attenuation is also largely eliminated by the addition of the waveguide bend. 
Proceeding of $3^{\text {rd }}$ International Conference on Engineering Mathematics and Physics Eph-1 irq

\section{REFERENCES}

1. Cisco ONS 15540 ESPx Planning Guide, pp 5-4, Ch. 5, (2004).

2. Zeng S.Z., Zhu D., Zhang B., and Lei W., "Design and Simulation of a Polymeric Variable Optical Atttenuator", in Proc. SPIE, Optical Fibers and Passive Components, pp 101-106, (2005).

3. Liu Q.Z., Jlang X.S., Yu L.S., Guan Z.F., Yu P.K.L., And Lau S.S., "A Novel Processing Technique to Fabricate Planar InGaAsP/InP Electroabsorption Waveguide Modulators", J. of Electronic Materials, Vol.24, No.8, pp 991-997, (1995).

4. Iodice M., Coppola G., Zaccuri R.C., and Rendina I., "Waveguide-vanishingbased optical modulator in embedded all-silicon structure", in Proc. SPIE, Optoelectronic Integration on Silicon II, pp 114-124, (2006).

5. Xiao G.Z., Zhang Z., and Grover C.P., "A Variable Optical Attenuator Based on a Straight Polymer-Silica Hybrid Channel Waveguide", IEEE Ph. Technol. Lett., Vol.16, No.11, pp 2511-2513, (2004).

6. Kowalczyk T.C., Finkelshtein I., Kouchnir M., Lee Y.C., Nguyen A.D., Vroom D., and Bischel W.K., "Polymeric Variable Optical Attenuator Array", Technical Digest. Summaries of papers presented at the Conference on Lasers and Electro-Optics, pp 17-18, (2001).

7. Veldhuis G.J., Krommendijk F.N., and Lambeck P.V., "Integrated optic intensity modulator based on a bent channel waveguide", Optics Communications, Vol.168, pp 481-491, (1999).

8. Tolstikhin V., Densmore A., Pimenov K., Logvin Y., Wu F., Laframboise S., and Grabtchak S., "Monolithically Integrated Optical Channel Monitor for DWDM Transmission Systems", J. Light. Technol., Vol.22, No.1, pp 145-153, (2004).

9. Koren U., Miller B.I., Young M.G., Koch T.L., Jopson R.M., Gnauck A.H., Evankow J.D., and Chien M., "High frequency modulation of strained layer multiple quantum well optical amplifiers", Electron. Lett., Vol.27, No.1, pp 62-64, (1991).

10. Tomjenovic-Hanic S. and Krolikowski W., "New design for a variable optical attenuator based on a bent channel waveguide", Appl. Phys. B, Vol.77, pp 19-23, (2003).

11. Bennett B.R., Soref R.A., and Delalamo J.A., "Carrier Induced Change in Refractive Index of InP, GaAs, and InGaAsP", IEEE J. Quantum Electron., Vol.26, No.1, pp 113-122, (1990).

12. Barrios P., Janz S., Delage A., Golub I., McKinnon R., Poole P.J., Abdalla S., Celo D., Ng S., Smy T.J., and Syrett B.A., "Digital optical switches with reconfigurable output waveguide branches in InP", Proc. SPIE: Integrated Optical Devices, Nanostructures, and Displays, pp 751-754, (2004). 
Proceeding of $3^{\text {rd }}$ International Conference on Engineering Mathematics and Physics Eph-1 $\quad$ \&.

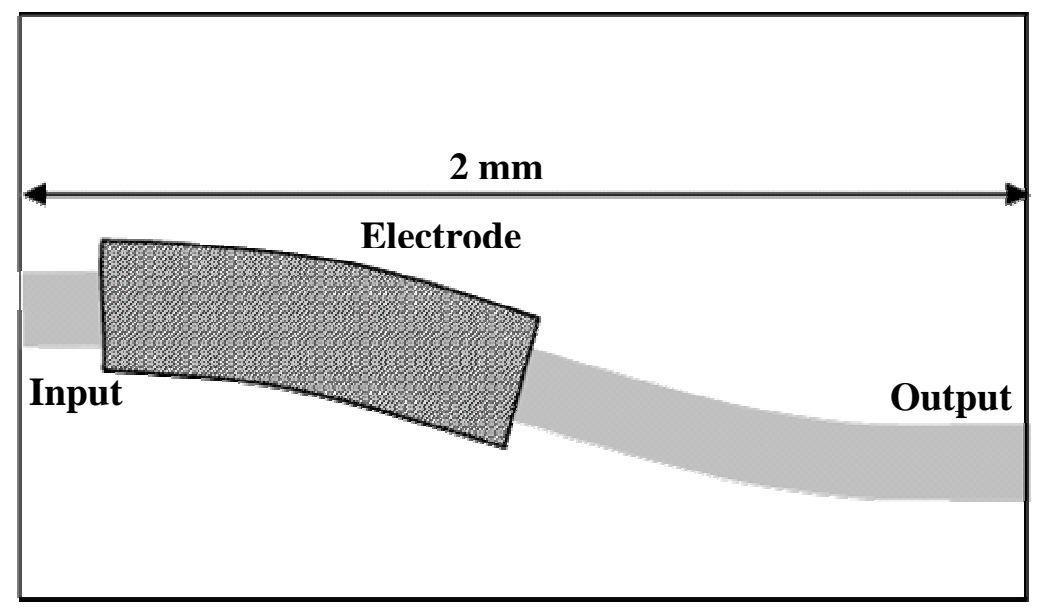

Fig. 1. Configuration of the proposed bend loss attenuator 




Fig. 2. Layer structure of the optical attenuator 


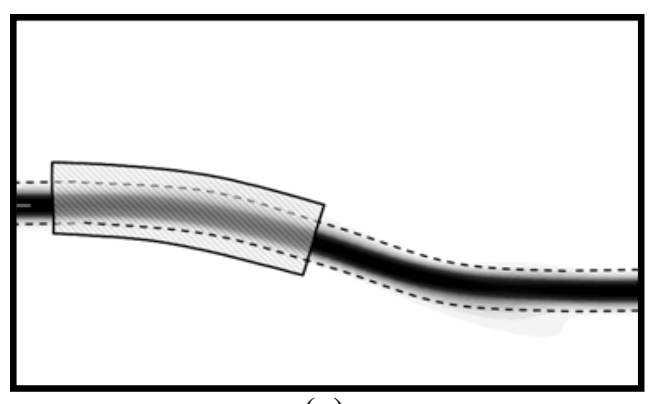

(a)

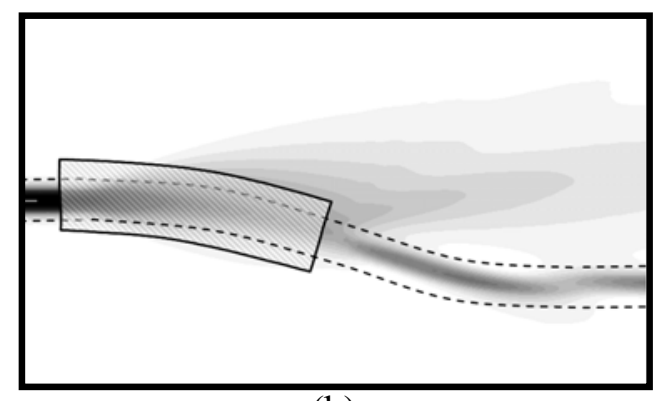

(b)

Fig. 3. BPM simulation results of a bend loss attenuator with input at the left and output at the right (a) Results of propagation through the unbiased device (b) Results of propagation through forward biased device 


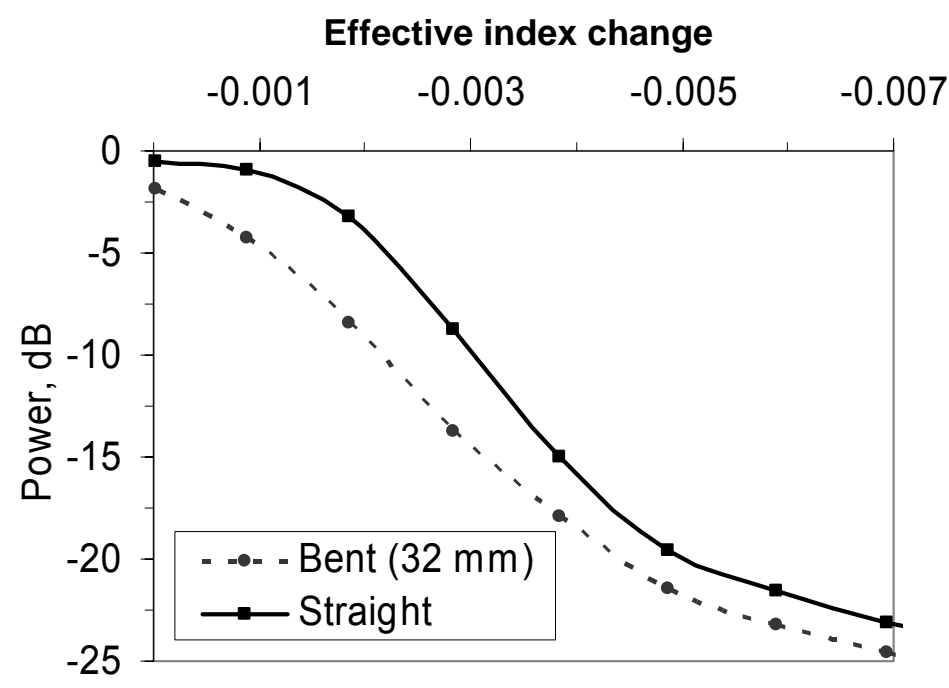

Fig. 4. BPM results for straight and bent waveguide attenuators. The calculation is for TE polarization and includes carrier induced absorption losses 


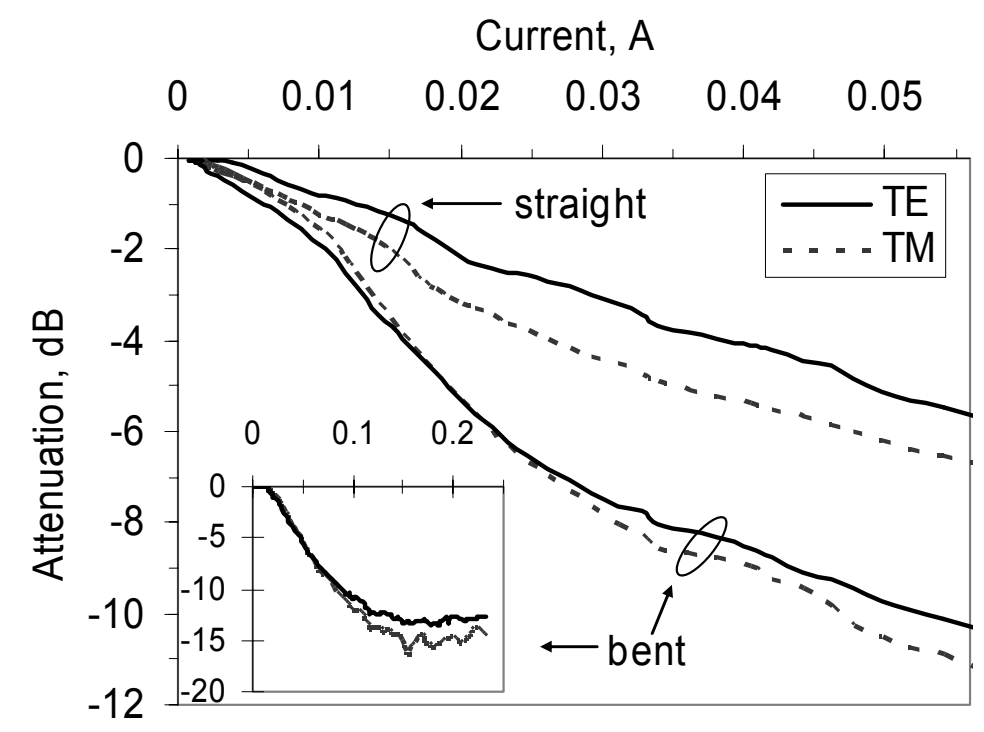

Fig. 5. Experimental results for straight and bent waveguide attenuators. The inset shows the bent waveguide data plotted over a larger range. 